\title{
Rassismus und Polizei
}

\section{Joachim Kersten}

\begin{abstract}
$\Lambda$ ngesichts der Vorfälle im »französischen Herbst « 2005 wurden gegen die Polizei des Nachbarlands Rassismusvorwürfe laut. Die Beamten seien bei den gewalttätigen Auseinandersetzungen nicht nur gegen die wenig »ohnmächtig « erscheinende Wut von »Halbstarken«, Chaoten und Unruhestiftern vorgegangen. Zur Sache gegangen wäre es zwischen einer praktisch rein weißen Polizei (männlichen Geschlechts) als Teil der französischen Mehrheitsgesellschaft und den dunkelhäutigen Angehörigen der nachwachsenden Generation diskriminierter ethnischer Minderheiten (gleichfalls männlich). Letztere haben trotz Schulabschluss und perfektem Französisch wegen Nachnamen und Hautfarbe keine Chance auf Job und Status. Die auch in unseren Fernsehnachrichten gezeigten Videoaufnahmen (Polizisten treten auf einen am Boden liegenden Zivilisten ein) ließen Erinnerungen an die Amateuraufzeichnungen des Rodney King Skandals in Los Angeles aufkommen, die zu Rassismus Vorwürfen gegen das Police Department von Los Angeles führten. Anders als in den USA wurden im Nachbarland die betreffenden Polizisten strafrechtlich zügig zur Verantwortung gezogen.
\end{abstract}

Amateuraufnahmen dokumentierten auch wie mehrere französische Polizisten die dunkelhäutige Ex-Weltmeisterin im Weitsprung, Eunice Barber, auf die Motorhaube ihres Autos drückten, sie mehrfach zu Boden zwangen, bevor sie über Nacht in Gewahrsam genommen wurde. Offi- zieller Grund: ein Verkehrsvergehen, inoffizielle Vermutung: Rassismus. Interner Polizeijargon bei U.S.-Polizisten für diese Art von Personenkontrolle und Vorgehen ist DWB. DWD heißt: Driving while drunk (Trunkenheitsfahrt), DWB, driving while black. »Schwarzfahrerkontrolle« wäre die freie Übersetzung.

Es gab in den letzten Jahrzehnten nach Vorfällen in unseren Städten gleichfalls Rassismusvorwürfe gegen Polizisten. Der Hamburger Polizeiskandal (es ging dabei um den Umgang mit dunkelhäutigen jungen Männern, die im Umfeld des Bahnhofs mit illegalen Drogen handelten) führte zu entsprechender Aufmerksamkeit von Medien und Politik. Die in der Folge durchgeführte Untersuchung der Polizeiführungsakademie Münster/ Universität Trier legte zwar keine verbreitete strukturelle Anfälligkeit für fremdenfeindliche und rassistische Haltungen bei den deutschen Polizeien offen. Dieser Studie fehlte jedoch ein Untersuchungsteil, mit dem solche Praktiken überhaupt hätten festgestellt werden können. Kursorische Ausflüge der damaligen Forscher ins polizeiliche Alltagsgeschäft in Problemzonen der Strafverfolgung konnten solche Instrumente nicht ersetzen. Die Frage nach dem Umgang der deutschen Polizei mit Fremdenfeindlichkeit und Rassismus bedarf letztlich weiterer sozialwissenschaftlicher Forschung.
Der Soziologiedozentin Astrid Jacobsen geht es dementsprechend um die Frage, was der Nutzen des Soziologieunterrichts für angehende Kommissarinnen und Kommissare sein kann. Sind die Sozialwissenschaften in der Ausbildung für das mittlere und höhere Management der Polizei wirklich weitgehend entbehrlich? Können die zu Wissenschaften ernannten Fächer der Polizeilehre diese Lücke mittlerweile umstandslos füllen wie einige im Polizeimanagement argumentieren? Oder ist die Kluft zwischen Programm und Wirklichkeit im Polizeialltag eine Folge des Abbaus sozialwissenschaftlicher Expertise? In den polizeilichen Publikationsorganen wird beispielsweise von der längst gelungenen Integration von Frauen in den polizeilichen Dienstalltag geschrieben. Die Forschung kommt zu anderen, eher entgegen gesetzten Ergebnissen. Wie sieht es mit der Integration von Minderheiten in diesen Arbeitsalltag aus? Dieser Frage widmet sich der zweite Beitrag. Sind innerhalb des Personals der deutschen Polizei Rassismus und Diskriminierung gegenüber Polizeiangehörigen mit anderer Hautfarbe an der Tagesordnung? Wie werden solche Haltungen geäußert, wie werden sie sanktioniert? In der internationalen Polizeiforschung wird in diesem Kontext vom »institutionellen Rassismus« (Bland et al. 1999; Zhao/Lovrich 1998) gesprochen. Gibt es diesen Rassismus bei den deutschen Ordnungshütern?

\section{Neugier braucht Methode}

\section{Soziologie in der Polizeiausbildung - ein Plädoyer}

W itze über Soziologen? In ihrer ersten Soziologievorlesung schütteln die Studierenden der Hochschule für Polizei den Kopf. Wie es mit Vorurteilen sei, ist meine nächste Frage. Zögerlich am Anfang, dann mit zunehmendem Eifer tauchen Bilder von Wollpulliträgern, Stuhlkreissitzern, Demonstranten, Haschkonsumenten, Linken, Bauwagenbewohnern, Feministinnen, Steinestreichlern samt ihren Schwestern und Brüdern auf.

Eine derartige »Einladung « in die Soziologie für Polizisten hat neben dem - durchaus pro- duktiven - Unterhaltungswert mindestens zwei Funktionen: Die Unsicherheit, die - anders als in den Fächern Recht und Kriminalistik - über die Soziologie herrscht, wird thematisiert und damit als Einstellung ernst genommen. Darüber hinaus ist die Sammlung von Wissen zu einem neuen, unbekannten Gegenstand schon selbst eine soziologische Methode: Durch die Fixierung von Bildern, Assoziationen und vielleicht auch Abneigungen kann das vorhandene, für selbstverständlich gehaltene Wissen über etwas vorübergehend konserviert und somit Raum für die Entdeckung neuen Wissens geschaffen werden. ${ }^{1}$
Astrid Jacobsen

Mit dem letzten Argument ist schon der Auftrag des Faches Soziologie für Polizisten umrissen, für den dieser Artikel argumentiert. Das Potential der Soziologie für die Polizei liegt in der Erweiterung des professionellen Blickes durch die soziologische Methode der systematischen Beschreibung und Analyse - und weniger in der Anwendung eines spezifisch soziologischen Fachjargons oder bestimmter soziologischer Theorien. Das »soziologische Denktraining «, das Polizisten befähigt, neue Perspektiven auf bekannte und unbekannte Phänomene zu entwickeln, zielt auf eine Qualifizierung der Polizeipraxis. 